\title{
Development of Advanced Control Design Software for Researchers and Engineers
}

\author{
Gary J. Balas; Andy Packard! John C. Doyleł Keith Glover’̣ and Roy Smith! \\ MUSYN INC. \\ 1009 Fifth St. SE \\ Minneapolis, MN $\mathbf{5 5 4 1 4}$ \\ 612.378.1742 415.704 .8730
}

\section{Introduction}

This paper gives a brief description of The $\mu$ Analysis and Synthesis Toolbox ( $\mu$-Tools), an advanced control design toolbox to be used in conjunction with MATLAB. $\mu$-Tools introduces CONSTANT, SYSTEM, and VARYING matrix types and over a hundred commands to manipulate them, including $H_{\infty}$ optimal control and $\mu$ analysis and synthesis functions. The VARYING type allows for matrices which are functions of an independent variable, and the SYSTEM type is a packed system matrix. The MATLAB implementation of these data types is described briefly in Section 2. A brief synopsis of the $\mu$-Tools functions are listed in Section 4. As an example of their use, the following commands implement the first $D-K$ iteration of $\mu$-synthesis for a SYSTEM matrix sys, using the $\mu$-Tools commands hinfsyn, frsp, au, musynfit, mult, ainv, starp, and vplot. A slightly more detailed example of a $\mu$-synthesis design is given in Section 5.

\> minfo(sys)

system: 8 states 6 outputs 6 inputs

\blk_struct $=[22 ; 22] ;$ nmeas $=2 ;$ ncont $=2$;

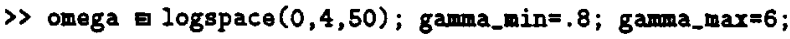

\> tolerancer.05;

\} [ k 1 , g 1 , g f 1 ] = \ldots

hinfsyn (sys, nmeas, ncont, gama_min, gama_max, tolerance);

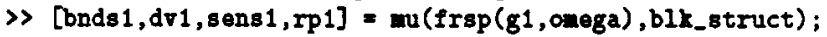
> [dsysl, dsysr] e...

musynfit ('1st_iter', dv1, sens1,b1r_struct,nmeas,ncont):

\sys2 = mult (dsysi, sys, ninv(dsjsr));

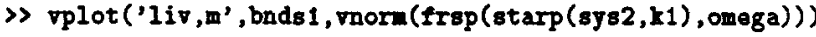

$\mu$-Tools has undergone several years of refinement in short courses and at various test-site locations. It is based on experience over a ten-year period with MATLAB derived control toolboxes as well as the HoneyX system developed at Honeywell's System and Research Center, where the most extensive applications of the methods provided by $\mu$-Tools has taken place. Beta test sites for the $\mu$-Tools software include Honeywell, McDonnell Douglas, and Philips. The software package has also been used in graduate controls classes at Caltech, Georgia Tech, U. C. Berkeley, and the University of Minnesota, and a textbook based on the toolbox is in preparation.

$\mu$-Tools is a unique software package in a number of respects. The most obvious feature is the introduction of control-specific data structures into the proven MATLAB environment. Perhaps more importantly, $\mu$-Tools represents the cutting edge in making advanced control theory available both to the researcher and the applications engineer. It will continue to be an outlet for the authors' theoretical research results, and its ongoing use at key industry and government centers will insure that it has contact with the most advanced control applications. The $\mu$-Tools authors have a strong commitment to applications and the development of theory with relevance to engineering design. Extensions under development include additional model reduction methods, analysis of systems with mixed complex and real parametric uncertainty, model validation and system identification for robust control, as well as several additional case studies.

\section{The Data Structures}

$\mu$-Tools represents systems (either in state-space form or as frequency dependent input/output data) as single data entries, providing all of the information about a system in a single MATLAB variable. In addition, the $\mu$-Tools functions which return a single variable can be nested, allowing you to build complex operations out of a few nested operations.

\subsection{SYSTEM Matrices}

Standard systems $\left[\begin{array}{c|c}A & B \\ \hline C & D\end{array}\right]$ are represented in $\mu$-Tools by a single MATLAB data structure, referred to as a SYSTEM matrix. The actual format of the data storage is

$$
\left[\begin{array}{rrr}
A & B & n x \\
C & D & 0 \\
0 & -I n f
\end{array}\right],
$$

where the zeros correspond to a row or column of zeros. The -Inf in the lower right corner indicates to the $\mu$-Tools functions that the matrix is a SYSTEM matrix. The number of states, $\mathrm{nr}$, uniquely determines the partitioning of the rest of the data.

\subsection{VARYING Matrices}

Matrix-valued functions of a single, independent real variable are represented in $\mu$-Tools with a data structure called a VARYING matrix. Frequency response and time responses of systems are the most common use for this type of structure, but other uses are possible. For example, a system which depended on a parameter could be represented as a VARYING matrix with the parameter as the independent variable and the matrix-valued function actually being a SYSTEM. Similarly, one can have VARYING matrices of VARYING matrices, and so on.

Consider a matrix valued function of a single real variable $G$ : $\mathbf{R} \rightarrow \mathbf{C}^{\mathbf{n} \times m}$, evaluated at $N$ discrete values of $x \in \mathbf{R}$, call them $x_{1}, x_{2}, \ldots, x_{N}$. Let $G_{i} \in C^{n \times m}$ be defined as $G\left(x_{i}\right)$. Then, in $\mu$-Tools, the actual data representation of the function $G$ is a MATLAB matrix with $n \cdot N+1$ rows, and $m+1$ columns, as shown below:

\footnotetext{
"Dept. of Aerospace Engineering and Mechanics, Univeraity of Minnecots

tDept. of Mechnnical Engineering, Univensity of California, Berkeley

tDept. of Electrical Engineering, Californis Intitute of Technology

Dept of Engineering, Cambridse Univercity, Ensland

Dept. of Electrical Engineering, Univernity of California, Sante Barbara
} 


$$
\left[\begin{array}{cc}
{\left[G_{1}\right]} & x_{1} \\
\vdots & \vdots \\
{\left[G_{i}\right]} & x_{N} \\
\vdots & 0 \\
{\left[G_{N}\right]} & \vdots \\
0.0 N & \text { Int }
\end{array}\right]
$$

The Inf in lower right corner tags this as a VARYING matrix. The number just to the left of the Inf, in this example, $N$, indicates how many data points are represented, and then the first $N$ values in the rightmost column are the independent variables values. The function data consists of the matrices, which are stacked one on top of one another.

\subsection{CONSTANT Matrices}

If a MATLAB entity is neither a SYSTEM nor a VARYING matrix it is treated by $\mu$-Tools as a CONSTANT matrix. CONSTANT matrices can be arguments to functions which normally expect VARYING or SYSTEM matrix arguments.

\section{Plotting VARYING Matrices}

The function vplot plots VARYING matrices. The argaments for vplot are similar to MATLAB's plot command, with the exception that it is not necessary to specify the values for the $x$-axis. The $x$ axis data corresponds to the independent variables, which are already stored within each VARYING matrix. An important feature of vplot is its ability to plot multiple VARYING matrices on the same plot without having to have the same independent variables.

In the MATLAB plot command, different axis types are provided by different functions, $\log \log , 8 \sin \log x$, and others. vplot provides this capability by an optional string argument. The default is a linear/linear scale. The generic vplot function call looks like

vplot ('axistype', vmat 1, 'linetype', valat2,...).

The aristype argument, a character string, allows the specification of logarithmic or linear axes as well as: magnitude, log magnitude, and phase. There are also some control specific options: bode, nyq, and nic which specify Bode, Nyquist, and Nichols plots respectively. The linetype arguments are optional and are identical to those provided by MATLAB for the plot command.

\section{Commands Grouped by Function}

\begin{tabular}{|ll|}
\hline \multicolumn{1}{|c|}{ Standard Operations/ Basic Functions } \\
\hline abv & $\begin{array}{l}\text { Stack constant/varying/system matrices above one } \\
\text { another }\end{array}$ \\
cjt & $\begin{array}{l}\text { Conjugate transpose of varying/system matrices } \\
\text { crand }\end{array}$ \\
Complex random matrix generator \\
daug & $\begin{array}{l}\text { Diagonal augmentation of constant/varying/system } \\
\text { matrices }\end{array}$ \\
madd & Addition of constant/varying/system matrices \\
minv & Inverse of constant/varying/system matrices \\
mmult & Multiplication of constant/varying/system matrices \\
mscl & Scale (by a scalar) a system or varying matrix \\
msub & Subtraction of constant/varying/system matrices \\
sbs & Stack matrices next to one another \\
sclin & Scale system input \\
sclout & Scale system output \\
sel & Select rows/columns or outputs/inputs \\
starp & Redheffer star product \\
transp & Transpose of varying/system matrices \\
\hline
\end{tabular}

Matrix Information, Displey and Plotting

dranag Interactive moused-based sketch and fitting tool

ninfo Information on a matrix

mrinte Formatted printing of a matrix

rifd Display real, imaginary, frequency and damping data

seo Display varying/system matrices

seoiv Display independent variables of a varying matrix

seesys Formatted varying/system display

vplot Plot a varying matrix

vzoon Mouse driven axis selection of plot window

\section{Modeling Functions}

nd2sys Convert a SISO transfer function into a system matrix

pck Create a system from $(A, B, C, D)$

pss2sys Convert [A B;C D] into a $\mu$-Tbols system matrix

sys2pss Extract [A B; C D] from a system

sysic System interconnection program

unpck Extract state-space data $(\boldsymbol{A}, B, C, D)$ from a system

2p2sys Convert poles and zeros to a system matrix

\begin{tabular}{|ll|}
\hline & \multicolumn{1}{c|}{ System Matrix Functions } \\
\hline reordsys & Reorder states in a system matrix \\
samhld & Sample-hold approximation of a continuous system \\
spoles & Poles of system matrix \\
statecc & Apply a coordinate transformation \\
strans & Bidiagonal coordinate transformation \\
sysrand & Generate a random system matrix \\
szeros & Transmission zeros of a system matrix \\
tustin & Prewarped continuous to discrete transformation \\
\hline
\end{tabular}

\begin{tabular}{|c|c|}
\hline & Model Reduction Functions \\
\hline $\begin{array}{l}\text { hankar } \\
\text { sdecomp } \\
\text { sfrutbal } \\
\text { sfrutbld } \\
\text { sncfbal } \\
\text { srelbal } \\
\text { sresid } \\
\text { strunc } \\
\text { sysbal }\end{array}$ & $\begin{array}{l}\text { Optimal Hankel norm approximation of a system } \\
\text { Decompose a system matrix into two system matrices } \\
\text { Frequency weighted balanced realization of } \\
\text { a system matrix } \\
\text { Stable frequency weighted realization of } \\
\text { a system matrix } \\
\text { Balanced realization of coprime factors of a system } \\
\text { matrix } \\
\text { Stochastic balanced realization of a system matrix } \\
\text { Residualize states of a system matrix } \\
\text { Truncate states of a system matrix } \\
\text { Balanced realization of a system matrix }\end{array}$ \\
\hline
\end{tabular}

\begin{tabular}{|ll|}
\hline & System Response Functions \\
\hline cos_tr & Generate a cosine signal as a varying matrix \\
dtrsp & Discrete time response of a linear system \\
frsp & Frequency response of a system matrix \\
sin_tr & Generate a sine signal as a varying matrix \\
siggen & Generate a signal as a varying matrix \\
step_tr & Generate a step signal as a varying matrix \\
trsp & Time response of a linear system \\
\hline
\end{tabular}

\begin{tabular}{|ll|}
\hline & $H_{2}$ and $H_{\infty}$ Analysis and Synthesis Functions \\
\hline csord & Order complex Schur form matrices \\
h2norm & Calculate 2-norm of a stable, strictly proper system \\
h2syn & $H_{2}$ control design \\
hinffi & $H_{\infty}$ full information control design \\
hinfnorm & Calculate $\infty$-norm of a stable, proper system \\
hinfsyn & $H_{\infty}$ control design \\
prvara & Peak norm of varying matrix \\
ric_eig & Solve a Riccati equation via eigenvalue decomposition \\
ric_schr & Solve a Riccati equation via real Schur decomposition \\
\hline
\end{tabular}


Structured Singular Value $(\mu)$ Analysis and Synthesis

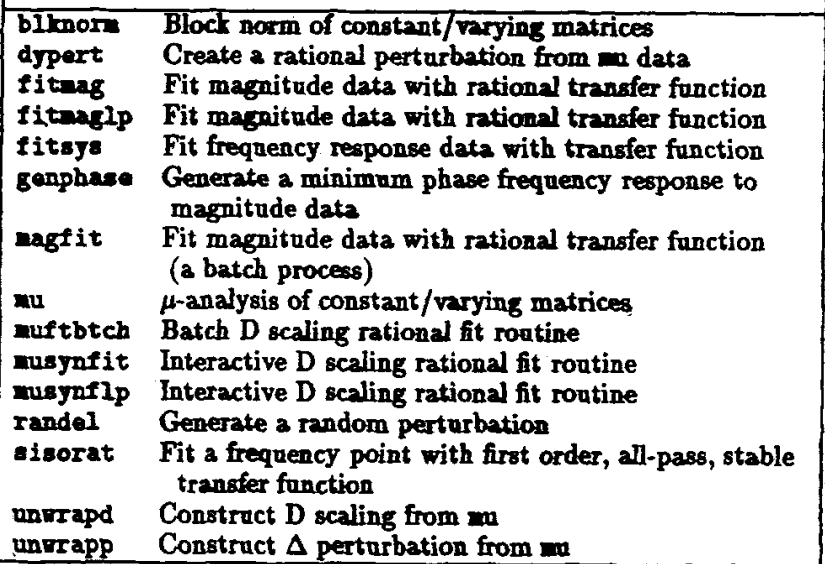

\begin{tabular}{|ll|}
\hline & Varying Matrix Manipulation \\
\hline getiv & Get the independent variable of a varying matrix \\
indrap & Compare the independent variable data \\
negangle & Calculate angle of elements between 0 and $-2 \pi$ \\
sc1iv & Scale the independent variable \\
sortiv & Sort the independent variable \\
tackon & String together varying matrices \\
var2con & Convert a varying matrix to a constant matrix \\
varyrand & Generate a random varying matrix \\
vdcmate & Decimate varying matrices \\
vinterp & Interpolate varying matrices \\
vpck & Pack a varying matrix \\
vapck & Unpack a varying matrix \\
rtract & Extract portions of a varying matrix \\
xtracti & Extract portions of a varying matrix \\
\hline
\end{tabular}

Many of the MATLAB matrix functions have analogous $\mu$-Tools functions which are identical on CONSTANT matrices, but operate on VARYING matrices on a matrix by matrix basis. These functions are

\begin{tabular}{|c|c|c|c|c|}
\hline $\begin{array}{l}\text { vabs } \\
\text { rerpan } \\
\text { vooly }\end{array}$ & $\begin{array}{l}\text { rceil } \\
\text { rfloor } \\
\text { rreond }\end{array}$ & $\begin{array}{l}\text { vdet } \\
\text { vint } \\
\text { rreal }\end{array}$ & $\begin{array}{l}\text { vdiag } \\
\text { vinag } \\
\text { voots }\end{array}$ & $\begin{array}{l}\text { reig } \\
\text { rnorm } \\
\text { rechmor }\end{array}$ \\
\hline
\end{tabular}

Additional $\mu$-Tools functions which extend MATLAB functionality to VARYING matrices are

rconj rejt rabe vfft rifte vldiv rrdiv vrho rtp

The functions roval and rebe perform a named operation on VARYING matrices. rebe performs MATLAB or user defined functions on the elements of a VARYING matrix (for example: sin, tan ...). reval can perform any function iacluding those with multiple input and output argements. rebe and reral allow the evaluation of any MATLAB matrix function on VARYING matrices. Several of these functions are illustrated below.

\begin{tabular}{|c|c|}
\hline MATLAB Matrix Function & $\mu$-Tools VARYING Function \\
\hline $\begin{array}{l}A+B+\ldots+B \\
A B ; A \mid B \\
A: B \\
i ; b \\
A: \\
\sin (A) \\
\operatorname{eax}(\operatorname{abs}(\operatorname{eig}(A))\end{array}$ & 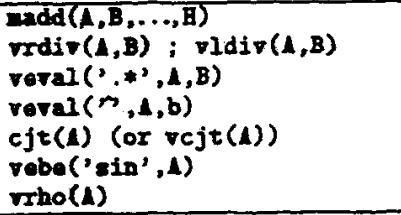 \\
\hline
\end{tabular}

\subsection{Special features}

$\mu$-Tools has several features that help make it user friendly. In addition to standard MATLAB help facilities, whenever a command is typed without any arguments, or with an incorrect number of arguments, a usage line is returned. For example, if the user has forgotten the inputs to au, simply type

\)

usage: [bnds, ron_d, sens, ron_p] $=$ m(natin, blk,opt)

For creating systems, in addition to nd2sys, zp2sys, sysic, and other programs that take command line arguments, dramag is an interactive mouse-based sketch and fitting tool. drannag creates a VARYING file of points at the locations where the mouse is clicked and a stable, minimum-phase system which approximately fits the points. This is particularly useful for creating weights for $\mu$-synthesis and candidate loopshapes for loopshaping designs.

The $\mu$-Tools commands for handling VARYING matrices are particularly useful for studying parametrized systems. Suppose we want to design a controller for the system

$$
p(s)=\frac{K s}{T s+1}
$$

where $K \in[1,10]$ and $T \in[.1,1]$. We can easily form a VARYING matrix with the 4 extreme corner systems with the following commands:

$\gg p 1=\operatorname{nd2sjs}(1,[11])$;

$>p 2=\operatorname{ascl}(p 1,10)$;

>pA $=\operatorname{nd2aja}(1,[.11])$;

>pP3 $=\operatorname{ascl}(p 4,10)$;

>>pstack $=\operatorname{vpck}([p 1 ; p 2 ; p 3 ; p 4 ; p 1],[1 ; 2 ; 3 ; 4 ; 1])$;

The VARYING matrix pstack contains 5 systems, with the last one a repeat of the first. This is done to provide good Nichols chart plots. The commands

$\gg c=\operatorname{mul}\left(\operatorname{nd2sys}\left(1,\left[\begin{array}{ll}1 & 0\end{array}\right), \operatorname{nd2sys}\left(\left[\begin{array}{ll}1 & 4\end{array}\right],\left[\begin{array}{ll}0 & 1\end{array}\right]\right), \ldots\right.\right.$

$\gg$ nd2ays $(1,[1 \theta-31]))$;

>cstack $=\operatorname{Tpck}([c ; c ; c ; c ; c],[1 ; 2 ; 3 ; 4 ; 1])$;

>lstack = roval ('ult', cstack, pstack);

form a 3rd order controller and multiply it by each plant to form a VARYING matrix of loop transfer functions. We can then take the frequency response and plot it in several ways.

\>Ivar = veral ('frsp', lstack, omega);

> Inic = veral ('vunpck', lvar);

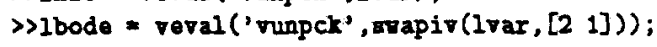

〉Ibode $=\operatorname{sel}($ lbode,1:4,1);

\vplot ('nic',1nic); grid;

\rplot ('bode', Ibode);
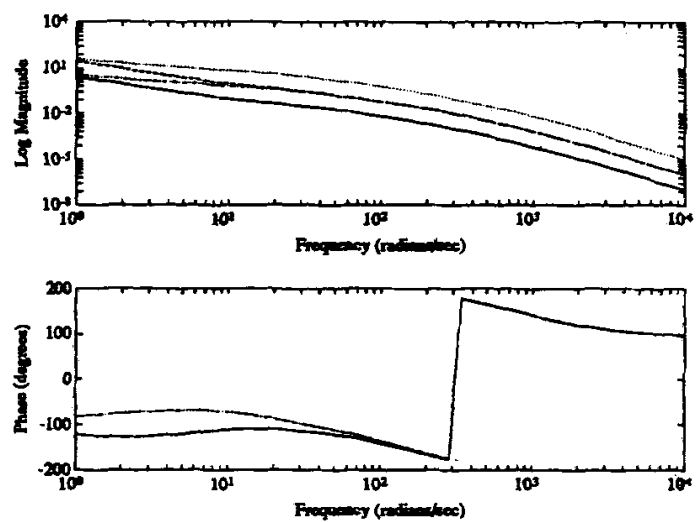


\section{Robust Performance Design Example}

This section contains an example of $\mu$-synthesis as applied to a paper design for the linearized pitch axis controller of an experimental highly maneuverable airplane, using a model taken from data for the HIMAT vehicle. Because of space constraints, only minimal engineering motivation will be give for this problem; it is presented only to illustrate the software. The $\mu$-Tools software comes with six M-files, himat $x 1$ through hinat $x 6$, which go through this example in greater detail. The problem is posed as a robust performance problem, with multiplicative plant uncertainty at the plant input and plant output weighted sensitivity function as the performance criterion. The design procedure involves several steps:

1. Specification of closed loop feedback structure.

2. Specification of model uncertainty and performance objectives in terms of frequency-dependent weighting matrices.

3. Construction of open-loop interconnection for control synthesis routines.

4. $\mathcal{H}_{\infty}$ optimal controller design for the open-loop interconnection.

5. Analysis of ROBUST PERFORMANCE properties of the resulting closed-loop systems using the structured singular value, $\mu$ ( $\mu$-analysis).

6. Use of frequency dependent similarity scalings, obtained in the $\mu$-analysis step, to scale the open loop interconnection, and redesign $\mathcal{H}_{\infty}$ controller (iterating on steps 5,6 , and 7 constitutes an approach to $\mu$-synthesis).

The state vector consists of the vehicle's basic rigid body variables:

$$
x^{T}=(\delta v, \alpha, q, \theta)
$$

representing the forward velocity, angle-of-attack, pitch rate, and pitch angle, respectively. The control inputs are the elevon $\left(\delta_{e}\right)$ and the canard $\left(\delta_{c}\right)$. The variables to be measured are $\alpha$ and $\theta$.

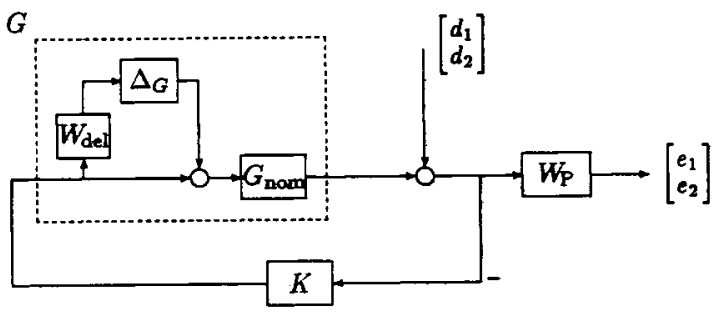

Figure 1: HIMAT Closed-loop Interconnection Structure

The control design objective is to design a stabilizing controller $K$ such that for all stable perturbations $\Delta_{G}(s)$, with $\left\|\Delta_{G}\right\|_{\infty}<1$, the perturbed closed-loop system remains stable, and the perturbed weighted sensitivity transfer function,

$$
S\left(\Delta_{G}\right):=W_{P}\left(I+P\left(I+\Delta_{G} W_{\mathrm{ded}}\right) K\right)^{-1}
$$

has $\left\|S\left(\Delta_{G}\right)\right\|_{\infty}<1$ for all such perturbations.

\subsection{Models and performance objectives}

Sources of uncertainty include uncertainty in the canard and the elevon actuators, in the forces and moments generated on the aircraft due to their deflections, uncertainty in the linear and angular accelerations produced by the aerodynamically generated forces and moments, and many others. In this example, we choose not to model the uncertainty in a detailed manner, but rather to lump all of these effects together into 1 full-block uncertainty at the input of a 4-state, nominal model of the aircraft rigid body. This nominal model has no (i.e., perfect) actuators and only quasi-steady dynamics. The nominal model for the airplane is loaded from the mutools/subs directory.

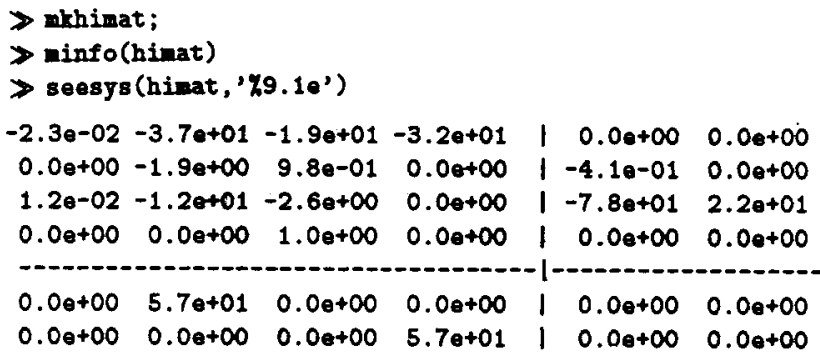

The partitioned matrix represents the $[A \quad B ; C$ D state space data For this example, $W_{\text {del }}:=v_{\text {del }}(s) I_{2}$, with $w_{\text {ded }}(s)=\frac{s 0(\rho+100)}{s+10000}$, anc $W_{p}(s)=w_{p}(s) I_{2}$, where $w_{p}(s)=\frac{0.5(s+3)}{s+0.03}$. The engineering motivatior for a performance specification like this would most naturally come from the desire to be able to have independent tracking of the angle of attack and pitch angle. $W_{\text {del }}$ can be formed as follows:

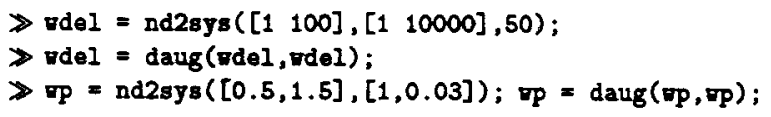

The phrases robust stability, nominal performance, and robust performance are used in this framework extensively. For this problem, they mean:

Nominal Performance:

$$
\left\|W_{p}\left(I+G_{\mathrm{nom}} K\right)^{-1}\right\|_{\infty}<1
$$

Robust Stability:

$$
\left\|W_{\mathrm{del}} K G_{\mathrm{nom}}\left(I+K G_{\mathrm{nom}}\right)^{-1}\right\|_{\infty}<1
$$

Robust Performance:

$$
\left\|W_{p}(I+G K)^{-1}\right\|_{\infty}<1
$$

is satisfied for every $G \in \mathcal{G}$. The property of robust performance is equivalent to a structured singular value test.

5.2 Building the open-loop interconnection with sysic A 6-input, 6-output SYSTEM matrix, himat_ic, (also referred to as $P(s))$

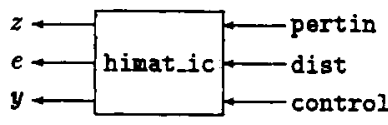

has internal structure shown in Figure 2.

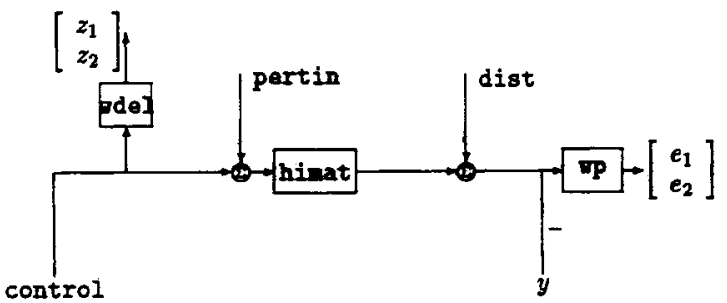

Figure 2: HIMAT Open-loop Interconnection Structure

This can be produced with nine MATLAB commands, listed below. The first 8 lines describe the various aspects of the interconnection, 
and may appear in any order. The last command, aysic, produces the final interconnection. The commands can be placed in an M-file, or executed at the command line.

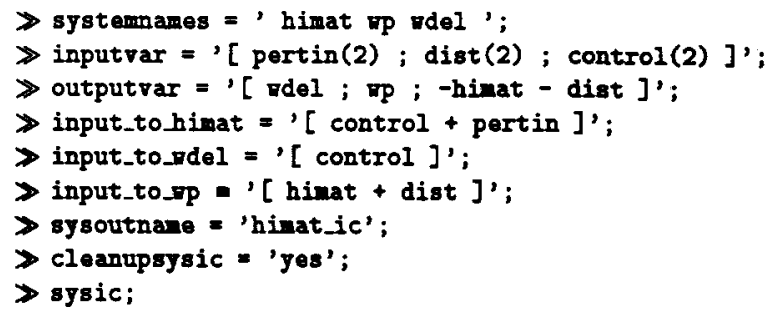

\section{$5.3 \mu$-synthesis and $D-K$ iteration}

For notational purposes, let $P(s)$ denote the transfer function of the six-input, six-output open-loop interconnection, himat_ic. Define a block structure $\Delta$ as

$$
\Delta:=\left\{\left[\begin{array}{cc}
\Delta_{1} & 0 \\
0 & \Delta_{2}
\end{array}\right]: \Delta_{1} \in C^{2 \times 2}, \Delta_{2} \in C^{2 \times 2}\right\} \subset \mathbf{C}^{4 \times 4} .
$$

The first block of this structured set corresponds to the full-block uncertainty $\Delta_{G}$ used in section 5.1 to model the uncertainty in the airplane's behavior. The second black, $\Delta_{2}$ is a fictitious uncertainty block, used to incorporate the $\mathcal{H}_{\infty}$ performance objectives on the weighted output sensitivity transfer function into the $\mu$-framework.

Recall that a stabilizing controller $K$ achieves closed-loop, robust performance if and only if for each frequency $\omega \in[0, \infty]$, the structured singular value

$$
\mu_{\Delta}\left[F_{l}(P, K)(j \omega)\right]<1
$$

Using the upper bound for $\mu$, (recall that in this case, 2 foll blocks, the upper bound is exactly equal to $\mu$ ) we can attempt to minimize the peak closed-loop $\mu$ value by posing the optimization problem

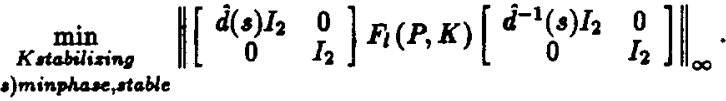

An approximate $\mu$-synthesis involves a sequence of minimizations, first over the controller variable $K$ (holding the $d$ variable fixed), and then over the $d$ variable (holding the $K$ variable fixed). This is often referred to as the $D-K$ iteration.

\section{4 $\mathcal{H}_{\infty}$ design on the open-loop interconnection}

In this section, we carry out the first step of the $D-K$ iteration, which is an $\mathcal{H}_{\infty}$ (sub) foptimal control design for the open-loop interconnection, himatic. In terms of the iteration, this amounts to holding the $d$ variable fixed (at 1 ), and minimizing the $\|\cdot\|_{\infty}$ norm of $F_{7}(P, K)$, over the controller variable $K$, as shown.
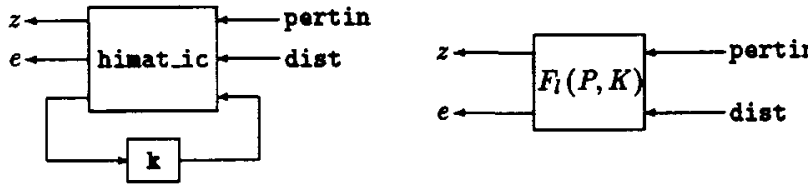

The function hinf syn designs a (sub)optimal $\mathcal{H}_{\infty}$ control law based on the open-loop interconnection structure provided. Syntax, input and output arguments to hinf syn are:

$$
>[\mathrm{k}, \mathrm{clp}]=\operatorname{hinfsyn}(\mathrm{p}, \text { nmeas, ncon,glor,ghigh, tol }) \text {; }
$$

The arguments are:

$$
\text { Inputs }
$$$$
\text { open-loop interconnection (SYSTEM matrix) p }
$$
number of measurements number of controls

lowrer bound on achievable norm

upper bound on achievable norm

absolute tolerance for bisection method

\section{ncons}

glor

ghigh

Outputs

controller (SYSTEM matrix)

closed-loop (SYSTEM matrix)

tol

$\mathbf{k}$

clp

In this example, the calling sequence is

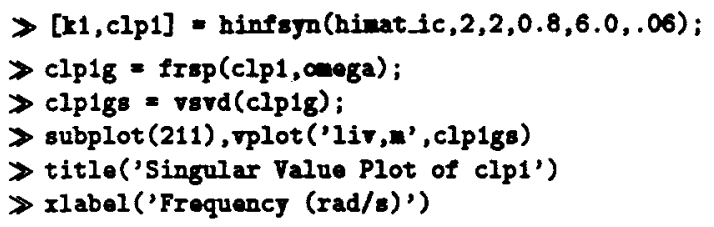

The two $2 \times 2$ transfer fonctions associated with robust stability and nominal performance may be evalnated separately, using the command sel.
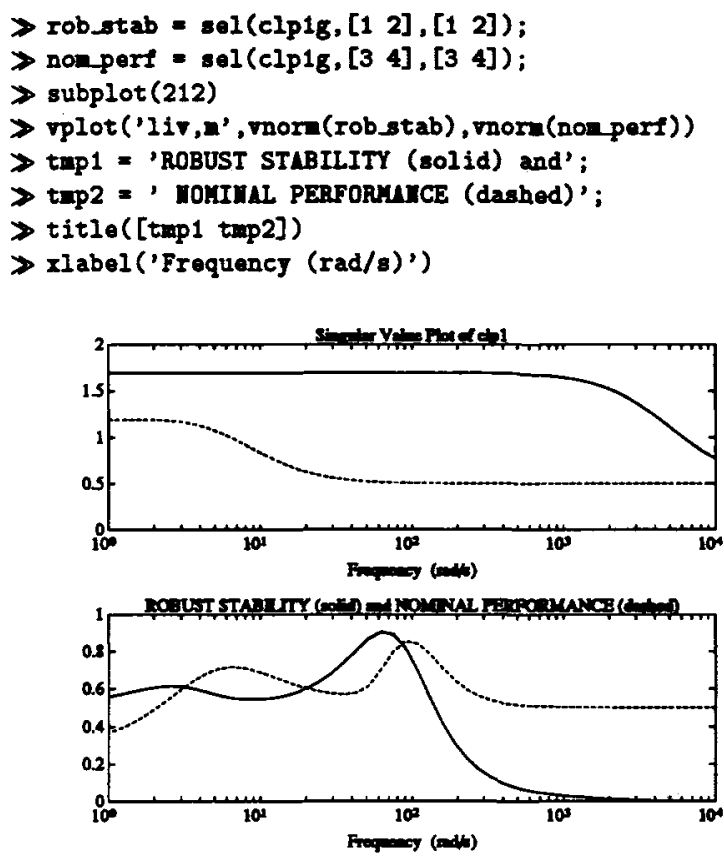

Note that the controlled system achieves both nominal performance and robust stability, since the peaks of the relevant singular value plots are less than 1.

\subsection{Assessing ROBUST PERFORMANCE with $\mu$}

The ROBUST PERFORMANCE properties of the closed-loop system can be analyzed using $\mu$-analysis. For a frequency domain $\mu$-analysis of ROBUST PERFORMANCE properties, the block structure should consist of a $2 \times 2$ uncertainty block, and a $2 \times 2$ performance block. We'll now make this calculation on the closed-loop from the $\mathcal{H}_{\infty}$ design. The density of the frequency points is increased from 50 to 100 in order to have smoother plots.

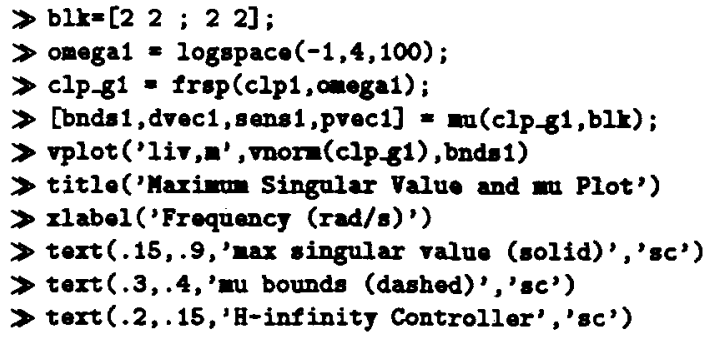




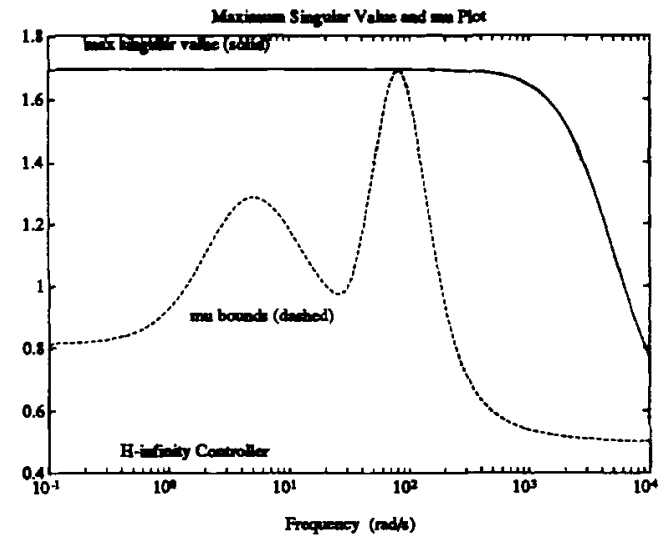

Figure 3: The dashed plot (which is actually two plots) is both the upper and lower bounds for $\mu_{\Delta}\left(F_{l}(P, K)(x \omega)\right)$.

Hence, the controlled system (from $\mathcal{H}_{\infty}$ ) does not achieve robust performance. This conclusion follows from the $\mu$ plot, which peaks to a value of 1.69 , at a frequency of $73.6 \mathrm{rad} / \mathrm{s}$. Since the upper and lower bounds are equal here, this is exact. The worst-case perturbattion can be constructed using dypert.

\subsection{One + iteration of $\mu$-synthesis}

Designing an $\mathcal{H}_{\infty}$ control law was the first computational step in $\mu$ synthesis. The second step involved a $\mu$ analysis on the closed-loop system. This calculation produces frequency dependent scaling matrices, called the $D$-scales. Using musyntit, the varying variables in the $D$-rcales can be fit (in magnitude) with stable, minimum phase rational functions and absorbed into the generalized plant for additional iterations. The syntax for musyneit is

$>$ [dsysL, dsysR $=\ldots$

musynfit (predsysL, Ddata, sens, blr, nmeas, ncont);

prodsysu: the rational $D$ scaling matrix from the previous iteration.

Ddata: the frequency varying $D$-scales from the previous $\mu$-analysis step. These are the "new" $D$ 's which need to be absorbed onto the existing scalings.

sens: the sensitivity variable from the previous $\mu$ analysis step.

blr: the uncertainty block structure, same as from the $\mu$-analysis.

nesas: number of measurements, same as in the $\mathcal{H}_{\infty}$ design.

ncon: number of controls, same as in the $\mathcal{H}_{\infty}$ design.

The output arguments are two system matrices, dsysL and dsysR. For the most part, these are the same thing - they are stable, minimum phase, block diagonal approximations to the product of the previous rational $D$, and the frequency varying $D$ (from $\mu$ calculation) which was the adjustment that was to be made to the rational $D$. The new interconnection structure is formed from the original unscaled hinat_ic together with dsysL and dsysR.

$>$ ner_ic $=$ mult (dsysL, himat_ic, ninv(dsysR));

musynf it runs interactively using the graphics window, but there is batch version as well. For the first iteration a 4th order fit was used. This increases the number of states in the open-loop interconnection structure by 16 states.

$>$ [dsysL1, dsysR1] = musynfit('first', drec1, sens 1, blk, 2,2);

$>$ mic1 $=$ mult (dsysL1, himat_ic,ninv(dsysR1));
This rational $D$ fitting concludes the first iteration of the $D-K$ iteration approach to $\mu$ synthesis. The process is repeated, beginning with a new $\mathcal{H}_{\infty}$ design on the generalized plant muic1. This open-loop interconnection, muic1, consists of the rational $D$-scalings dsysL 1 and dsysRi absorbed onto the original open-loop interconnection, himat_ic.

The second iteration of $D-K$ iteration is comprised of the following steps listed below. A third order fit was used for the D-scaling.

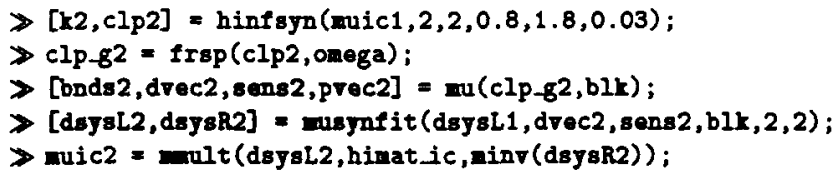

The third iteration of $D-K$ iteration is:
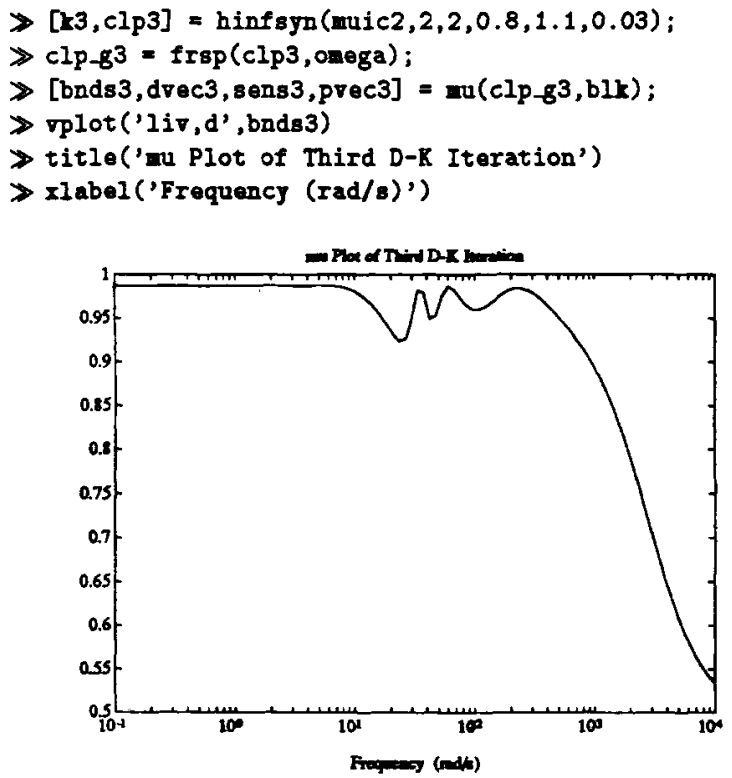

On the third iteration, the value of $\mu$ was less than 1 across frequency. This implies that robust performance was achieved for the closed loop system with controller $\mathbf{r} 3$. Following the steps above, one can carry on as many iterations as desired. In general, the $D-K$ iteration process is continued until either $\mu$ is less than 1 , indicating the robust performance has been achieved, or the peak $\mu$ value of the closed-loop system stops decreasing.

\section{Summary}

This paper provides a brief description of The $\mu$ Analysis and Synthesis Toolbox ( $\mu$-Tools), an advanced control design toolbox to be used in conjunction with MATLAB. 